\title{
LETTER
}

\section{Defining patient-ventilator asynchrony severity according to recurrence}

\author{
Kay Choong See ${ }^{1,2^{*}} \mathbb{D}$, Juliet Sahagun ${ }^{3}$ and Juvel Taculod ${ }^{3}$
}

(0) 2020 Springer-Verlag GmbH Germany, part of Springer Nature

Dear Editor,

Patient-ventilator asynchrony (PVA) is a mismatched interaction between the patient's needs and the ventilator-delivered breath. Types of PVA include trigger asynchrony (problem with mechanical inspiration), flow asynchrony (problem with inspiratory flow delivery) and cycling-off asynchrony (problem with timing of mechanical expiration). Almost all mechanically ventilated patients experience PVA [1], though poor clinical outcomes have only been associated with severe PVA. Some authors define severe PVA using proportion $(\geq 10 \%$ of breaths are asynchronous) [2], while others use clustering of PVA events [3]. However, these definitions do not allow ready selection of patients for personalized treatment. We therefore propose defining PVA severity based on recurrence and explored the association of recurrent PVA with clinical outcomes.

We studied patients who were intubated in the emergency department and directly admitted to the medical intensive care unit (ICU), from February 2017 to July 2017 (Figure E1, online ESM). Nurses titrated analgesia to achieve a Critical-Care Pain Observation Tool score of $0-2$ and sedation to achieve a Richmond Agitation Sedation Scale score -2 to 0 . Respiratory therapists also implemented a PVA protocol (reflecting our usual practice) for all mechanically ventilated patients upon ICU admission and twice daily (7 am, $7 \mathrm{pm}$ ), which involved bedside observation and management of PVA events for

\footnotetext{
*Correspondence: kay_choong_see@nuhs.edu.sg

${ }^{1}$ Division of Respiratory and Critical Care Medicine, University Medicine Cluster, National University Health System, 1 E Kent Ridge Road, NUHS

Tower Block Level 10, Singapore 119228, Singapore

Full author information is available at the end of the article
}

This work was performed at the National University Health System, Singapore. at least 2 min each time (Table E1, online ESM). During each PVA check, PVA was noted as a dichotomy (present versus absent) and was coded as present if the sum of asynchronous breaths exceeded 2 over 120 s. Recurrent asynchrony is defined as two or more PVA checks at two different times where asynchrony was coded as present. Logistic regression was used to examine the association of nonrecurrent and recurrent asynchrony with ICU/ hospital mortality, adjusting for any factors that were statistically significant on univariate analysis.

One hundred twenty patients were studied (age $64.8 \pm 12.5$ years, $45 / 37.5 \%$ female, APACHE II score $26.7 \pm 8.1,116 / 96.7 \%$ on volume assist control initially); 1635 episodes of PVA checks were performed for 120 patients (median seven checks per patient, interquartile range 3-18.5), of whom 35 (29.2\% of 120 patients) experienced 110 episodes of PVA. The most common PVA was double triggering (64 episodes/3.9\%), and the most common actions taken were to increase inspiratory flow, tidal volume or sedation (35-38 times, respectively) (Tables E2 and E3, online ESM). Presence of ARDS, use of nonvolume assist-control ventilation mode and use of dexmedetomidine were associated with asynchrony, though ventilation mode was only associated with nonrecurrent asynchrony. Recurrent asynchrony, but not nonrecurrent asynchrony, was associated with increased ICU and hospital mortality (Table 1 ).

The association of asynchrony recurrence with mortality suggests that it may be used to identify severe asynchrony. Using PVA recurrence as a severity criterion has several advantages compared to proportion or clustering of PVA events: It avoids the need for continuous monitoring, it can be done using simple bedside observation, and it can be applied prospectively to select patients for further treatment, e.g., neuromuscular blockade $[4,5]$. Nonetheless, given our single-center design, our proposed concept of recurrent

\section{望


Table 1 Characteristics and outcomes of patients with and without PVA

\begin{tabular}{|c|c|c|c|c|c|c|}
\hline Patient characteristics and outcomes & \multicolumn{2}{|c|}{ Patients without PVA } & \multicolumn{2}{|c|}{$\begin{array}{l}\text { Patients with nonrecurrent } \\
\text { PVA }\end{array}$} & \multicolumn{2}{|c|}{$\begin{array}{l}\text { Patients with recur- } \\
\text { rent PVA }\end{array}$} \\
\hline Number of patients & \multicolumn{2}{|l|}{85} & \multicolumn{2}{|l|}{18} & \multicolumn{2}{|l|}{17} \\
\hline Median number of asynchrony episodes & \multicolumn{2}{|l|}{ NA } & \multicolumn{2}{|l|}{1} & \multicolumn{2}{|l|}{3} \\
\hline IQR & \multicolumn{2}{|l|}{ NA } & \multicolumn{2}{|l|}{ NA } & \multicolumn{2}{|l|}{$2-5$} \\
\hline Range & \multicolumn{2}{|l|}{ NA } & \multicolumn{2}{|l|}{ NA } & \multicolumn{2}{|l|}{$2-21$} \\
\hline Age (years) & \multicolumn{2}{|l|}{$63.8 \pm 13.4$} & \multicolumn{2}{|l|}{$66.5 \pm 10.3$} & \multicolumn{2}{|l|}{$67.7 \pm 9.1$} \\
\hline Female (\%) & \multicolumn{2}{|l|}{$33(38.8)$} & \multicolumn{2}{|l|}{$7(38.9)$} & \multicolumn{2}{|l|}{$5(29.4)$} \\
\hline APACHE II score & $26.1 \pm 8.6$ & & $27.2 \pm 7.4$ & & $29.1 \pm 6.4$ & \\
\hline $\operatorname{ARDS}(\%)$ & $24(28.2)$ & & $6(33.3)$ & & $10(58.8)^{*}$ & \\
\hline Height (m) & $1.6 \pm 0.11$ & & $1.57 \pm 0.1$ & & $1.61 \pm 0.11$ & \\
\hline Weight (kg) & $63.5 \pm 17.3$ & & $61.2 \pm 13.8$ & & $64.8 \pm 17.5$ & \\
\hline Primary diagnosis (\%) & & & & & & \\
\hline Pneumonia & $25(29.4)$ & & $7(38.9)$ & & $9(52.9)$ & \\
\hline Other sepsis & $15(17.7)$ & & $3(16.7)$ & & $3(17.7)$ & \\
\hline COPD & $3(3.5)$ & & $0(0)$ & & $0(0)$ & \\
\hline Asthma & $3(3.5)$ & & $1(5.6)$ & & $1(5.9)$ & \\
\hline Stroke & $8(9.4)$ & & $3(16.7)$ & & $2(11.8)$ & \\
\hline Other $^{\mathrm{a}}$ & $31(36.5)$ & & $4(22.2)$ & & $2(11.8)$ & \\
\hline Comorbidity (\%) & & & & & & \\
\hline Diabetes mellitus & $36(42.4)$ & & $7(38.9)$ & & $4(23.5)$ & \\
\hline Hypertension & $48(56.5)$ & & $9(50)$ & & $11(64.7)$ & \\
\hline Ischemic heart disease & $18(21.2)$ & & $4(22.2)$ & & $3(17.7)$ & \\
\hline Chronic heart failure & $3(3.5)$ & & $1(5.6)$ & & $0(0)$ & \\
\hline Asthma & $7(8.2)$ & & $0(0)$ & & $2(11.8)$ & \\
\hline COPD & $4(4.7)$ & & $1(5.6)$ & & $0(0)$ & \\
\hline Chronic kidney disease & $17(20)$ & & $1(5.6)$ & & $3(17.7)$ & \\
\hline Chronic liver disease & $15(17.7)$ & & $3(16.7)$ & & $3(17.7)$ & \\
\hline Stroke & $3(3.5)$ & & $1(5.6)$ & & $0(0)$ & \\
\hline Cancer & $13(15.3)$ & & $2(11.1)$ & & $4(23.5)$ & \\
\hline ICU admission parameters & & & & & & \\
\hline Temperature (Celsius) & $36.9 \pm 1.2$ & & $36.7 \pm 1.3$ & & $36.8 \pm 1$ & \\
\hline Heart rate (beats/min) & $99 \pm 26$ & & $105 \pm 27$ & & $107 \pm 20$ & \\
\hline $\operatorname{MAP}(\mathrm{mmHg})$ & $94 \pm 23$ & & $91 \pm 24$ & & $97 \pm 17$ & \\
\hline Respiratory rate (/min) & $24 \pm 6$ & & $25 \pm 6$ & & $24 \pm 6$ & \\
\hline ICU admission ABG & & & & & & \\
\hline $\mathrm{pH}$ & $7.33 \pm 0.15$ & & $7.31 \pm 0.18$ & & $7.36 \pm 0.14$ & \\
\hline pCO2 (mmHg) & $42.0 \pm 19.2$ & & $34.0 \pm 8.6$ & & $46.4 \pm 23.9$ & \\
\hline Bicarbonate (mmol/L) & $20.8 \pm 6.1$ & & $18.8 \pm 7.3$ & & $23.5 \pm 4.9$ & \\
\hline Ventilation mode on ICU admission (\%) & & & & & & \\
\hline Volume assist control & $84(98.8)$ & & $16(88.9)^{*}$ & & $16(94.1)$ & \\
\hline Pressure assist control & $0(0)$ & & $2(11.1)$ & & $1(5.9)$ & \\
\hline Pressure support & $1(1.2)$ & & $0(0)$ & & $0(0)$ & \\
\hline Ventilation parameters on ICU admission ${ }^{b}$ & & $\mathrm{~N}^{\mathrm{e}}$ & & $\mathrm{N}^{\mathrm{e}}$ & & $\mathrm{N}^{\mathrm{e}}$ \\
\hline $\mathrm{FIO} 2$ & $0.68 \pm 0.16$ & 85 & $0.74 \pm 0.12$ & 18 & $0.69 \pm 0.2$ & 17 \\
\hline PEEP $(\mathrm{cmH} 2 \mathrm{O})$ & $5.8 \pm 1.7$ & 85 & $5.6 \pm 1.6$ & 18 & $6.2 \pm 2.2$ & 17 \\
\hline Tidal volume (ml/kg IBW) & $7.2 \pm 1.7$ & 73 & $7.9 \pm 2$ & 14 & $7 \pm 0.8$ & 12 \\
\hline Driving pressure $(\mathrm{cmH2O})$ & $11.7 \pm 4.7$ & 59 & $12.7 \pm 5.1$ & 13 & $11.1 \pm 5.8$ & 10 \\
\hline Analgesia/sedation use ${ }^{c}$ & & & & & & \\
\hline Fentanyl (\%) & $78(91.8)$ & & $17(94.4)$ & & $17(100)$ & \\
\hline Propofol (\%) & $75(88.2)$ & & $18(100)$ & & $17(100)$ & \\
\hline
\end{tabular}


Table 1 (continued)

\begin{tabular}{|c|c|c|c|}
\hline Patient characteristics and outcomes & Patients without PVA & $\begin{array}{l}\text { Patients with nonrecurrent } \\
\text { PVA }\end{array}$ & $\begin{array}{l}\text { Patients with recur- } \\
\text { rent PVA }\end{array}$ \\
\hline Midazolam (\%) & $5(5.9)$ & $1(5.6)$ & $1(5.9)$ \\
\hline Dexmedetomidine (\%) & $9(10.6)$ & $2(11.1)$ & $9(52.9)^{*}$ \\
\hline Median fluid balance at $24 \mathrm{~h}$ after ICU admission (IQR) (ml) & $669(105-1600)$ & $1415(503-2300)$ & $650(305-1333)$ \\
\hline Vasopressor use on ICU admission (\%) & $36(42.4)$ & $11(61.1)$ & $8(47.1)$ \\
\hline Median ventilator-free days through day 28 (IQR) & $25(23-26)$ & $23.5(21-25)^{*}$ & $18(10-23)^{*}$ \\
\hline Median sedation-free days through day 28 (IQR) & $26(25-27)$ & $25.5(24-27)$ & $22(18-26)^{*}$ \\
\hline Median ICU LOS (IQR) & $6(4-9)$ & $7.5(4-11)$ & $12(7-18)^{*}$ \\
\hline Median hospital LOS (IQR) & $14(8-31)$ & $12.5(8-35)^{*}$ & $17(12-28)$ \\
\hline ICU mortality (\%) & $10(11.8)$ & $5(27.8)$ & $7(41.2)^{*}$ \\
\hline Hospital mortality (\%) & $12(14.1)$ & $5(27.8)$ & $7(41.2)^{*}$ \\
\hline \multicolumn{4}{|l|}{ ICU mortality (OR, 95\% CI) } \\
\hline Unadjusted & Reference & $2.88(0.85-9.81)$ & $5.25(1.62-16.91)$ \\
\hline Adjusted $^{d}$ & Reference & $2.51(0.71-8.93)$ & $4.35(1.17-16.24)$ \\
\hline \multicolumn{4}{|l|}{ Hospital mortality (OR, 95\% Cl) } \\
\hline Unadjusted & Reference & $2.34(0.71-7.76)$ & $4.26(1.36-13.35)$ \\
\hline Adjusted $^{\mathrm{d}}$ & Reference & $2.07(0.6-7.17)$ & $3.81(1.05-13.82)$ \\
\hline
\end{tabular}

$\mathrm{ABG}$, arterial blood gas; $\mathrm{APACHE}$, acute physiology and chronic health evaluation; ARDS, acute respiratory distress syndrome; $\mathrm{BP}$, blood pressure; $\mathrm{Cl}$, confidence interval; COPD, chronic obstructive pulmonary disease; and IBW, IBW: ideal body weight. For males, IBW= $50 \mathrm{~kg}+2.3 \mathrm{~kg}$ for each increment of $2.54 \mathrm{~cm}$ ( 1 inch) in length over $152.4 \mathrm{~cm}$ (5 feet). For females, IBW $=45.5 \mathrm{~kg}+2.3 \mathrm{~kg}$ for each increment of $2.54 \mathrm{~cm}$ ( 1 inch) in length over $152.4 \mathrm{~cm}(5 \mathrm{feet}) ; \mathrm{ICU}$, intensive care unit; IQR, interquartile range; LOS, length-of-stay; OR, odds ratio; NA, not applicable; and PVA, patient-ventilator asynchrony

* $P<0.05$, compared to patients without PVA

a Includes myocardial infarction, bleeding gastrointestinal tract, status epilepticus, drug overdose, pulmonary embolism, diabetic ketoacidosis

b Done immediately after first application of the synchrony protocol

c Medication utilization over the course of each patient's ICU stay. A patient could be on more than one of these medications

$\mathrm{d}$ Adjusted for the presence of acute respiratory distress syndrome, use of volume assist-control mode of ventilation and use of dexmedetomidine

e Discrepancy in numbers between FIO2 and PEEP vs. tidal volume was because the tidal volume values were not recorded on admission for some patients. Discrepancy in numbers between FIO2 and PEEP vs. driving pressure was because plateau pressure was not measured on admission for some patients

asynchrony as a severity marker for PVA requires broader validation.

\section{Electronic supplementary material}

The online version of this article (https://doi.org/10.1007/s00134-020-05974-y) contains supplementary material, which is available to authorized users.

\section{Author details}

${ }^{1}$ Division of Respiratory and Critical Care Medicine, University Medicine Cluster, National University Health System, 1 E Kent Ridge Road, NUHS Tower Block Level 10, Singapore 119228, Singapore. ${ }^{2}$ Department of Medicine, Yong Loo Lin School of Medicine, National University of Singapore, Singapore, Singapore. ${ }^{3}$ Division of Critical Care - Respiratory Therapy, National University Hospital, Singapore, Singapore

\section{Acknowledgements}

The authors would like to thank the physicians, nurses and respiratory therapists who have cared for our patients.

\section{Authors' contributions}

KCS was involved in study concept, design and drafting of manuscript. All authors conducted the study and were involved in data analysis and interpretation and critical revision of the manuscript for important intellectual content. All authors had full access to all of the data in the study and took responsibility for the integrity of the data and the accuracy of the data analysis.
Funding

No funding was required for this study.

Availability of data and material

The data that support the findings of this study are available from the corresponding author, $\mathrm{KCS}$, upon reasonable request.

\section{Compliance with ethical standards}

\section{Conflicts of interest}

KCS has received honoraria and travel support from Medtronic. JS and JT have no conflicts of interest to declare.

\section{Consent for publication}

Not applicable.

\section{Ethics approval}

Our Ethics Review Board (National Healthcare Group Domain-Specific Review Board) approved the study (approval number DSRB 2018/00223). Given the observational study design, the need for patient consent was waived.

\section{Publisher's Note}

Springer Nature remains neutral with regard to jurisdictional claims in published maps and institutional affiliations. 
Accepted: 17 February 2020

Published online: 24 February 2020

\section{References}

1. Thille AW, Rodriguez P, Cabello B, Lellouche F, Brochard L (2006) Patientventilator asynchrony during assisted mechanical ventilation. Intensive Care Med 32:1515-1522

2. Blanch L, Villagra A, Sales B, Montanya J, Lucangelo U, Lujan M, GarciaEsquirol O, Chacon E, Estruga A, Oliva JC, Hernandez-Abadia A, Albaiceta GM, Fernandez-Mondejar E, Fernandez R, Lopez-Aguilar J, Villar J, Murias G, Kacmarek RM (2015) Asynchronies during mechanical ventilation are associated with mortality. Intensive Care Med 41:633-641
3. Vaporidi K, Babalis D, Chytas A, Lilitsis E, Kondili E, Amargianitakis V, Chouvarda I, Maglaveras N, Georgopoulos D (2017) Clusters of ineffective efforts during mechanical ventilation: impact on outcome. Intensive Care Med 43:184-191

4. National Heart L, Blood Institute PCTN, Moss M, Huang DT, Brower RG Ferguson ND, Ginde AA, Gong MN, Grissom CK, Gundel S, Hayden D, Hite RD, Hou PC, Hough CL, Iwashyna TJ, Khan A, Liu KD, Talmor D, Thompson BT, Ulysse CA, Yealy DM, Angus DC (2019) Early neuromuscular blockade in the acute respiratory distress syndrome. N Engl J Med 380:1997-2008

5. Papazian L, Forel JM, Gacouin A, Penot-Ragon C, Perrin G, Loundou A, Jaber S, Arnal JM, Perez D, Seghboyan JM, Constantin JM, Courant P, Lefrant JY, Guerin C, Prat G, Morange S, Roch A, Investigators AS (2010) Neuromuscular blockers in early acute respiratory distress syndrome. N Engl J Med 363:1107-1116 